\title{
Dynamics of a New Rumor Propagation Model with the Spread of Truth
}

\author{
Yujiang Liu*, Chunmei Zeng, Youquan Luo \\ Key Laboratory of Jiangxi Province for Numerical Simulation and Emulation Techniques, Gannan Normal University, \\ Ganzhou, China \\ Email: *gnsylyj@126.com
}

How to cite this paper: Liu, Y.J., Zeng, C.M. and Luo, Y.Q. (2018) Dynamics of a New Rumor Propagation Model with the Spread of Truth. Applied Mathematics, 9, 536-549.

https://doi.org/10.4236/am.2018.95038

Received: April 15, 2018

Accepted: May 27, 2018

Published: May 30, 2018

Copyright (C) 2018 by authors and Scientific Research Publishing Inc. This work is licensed under the Creative Commons Attribution International License (CC BY 4.0).

http://creativecommons.org/licenses/by/4.0/

\begin{abstract}
A mathematical model described the propagation of information including rumor and truth presented and its properties investigated. We explored exists of the equilibria, local stability and global asymptotical stability, and obtained the propagation threshold of rumor spreading. Numerical simulation is shown to demonstrate our results. Uncertainty and sensitivity analysis shows the importance of the parameters in our model.
\end{abstract}

\section{Keywords}

Rumor Propagation, Counter-Rumor, Sensitivity Analysis, Local Bistable, Global Stability

\section{Introduction}

Rumors, as daily events have been occurred. With the development of new media such as Internet, mobile phone has broadened the public access to information, optimized the circulation of information, but also exacerbated the spread of rumors. In emergency communication, financial markets fluctuation and all kinds of contagions, rumors play an important role [1] [2] [3]. Therefore, understanding the propagation of rumors and how to control it effectively is a very meaningful topic.

The dynamic behavior of the rumor spread has a great similarity with the spread of infectious diseases. Epidemic models have been developed by many researchers [4]-[14]. The classical model of rumor spreading was introduced by Daley and Kendal [15] [16]. In their model, the population is subdivided into three groups, the ignorant who know nothing about the rumor, the spreader who have heard the rumor and spread it again, the Stifler, who have heard the 
rumor but lose interesting with it and have ceased to spread it. Since then, many scholars worked on improving the model [17] [18]. Rumor spreading is under a close relationship with the network topology. Zanette [19] [20] examined the rumor spreading dynamics on small-world networks and obtained a critical threshold of rumor spreading. Some scholars developed applications of the stochastic Maki-Thompson model on scale-free networks [21] [22] [23] [24]. In recent years, numerous researchers have been studying the influence of psychological factors, such as memory, suspicion, forgetting and other factors of the spread of rumors [25]-[31]. Kawachi [25] studied the effect of the Stifler's memory on the spread of rumors, and when he was remembered for the rumors, it was the first to judge the true or false of the rumor. Zhao [30] discussed the influence of forgetting and remembering on the final size of the rumor. He suggested that as the forgetting rate increases, the final size of the rumor decreases, reversely, the bigger remembering rate makes the final size of the rumor larger. Wang [31] considered two different spreaders to spread two rumors individually and found that the spreading of one rumor inhibits the spreading of another rumor. Although above models are on the rumor spreading research made a significant contribution, these models are not reflecting the impact on the government counter-rumor. In the era of big data, the relevant government departments can take a variety of channels to eliminate or limit the spread of rumors. For example, after the rumor, they can through SMS (Short Message Service), government radio, broadcast, official microblog and counter-rumor sites to the society announced the truth of the incident, to eliminate unnecessary suspicion, alleviate people's anxiety and panic caused by the rumor spread and eliminate or reduce the loss of rumors to us. The rumor model takes into account the counter-rumor conforms to the rumors spread dynamic in today's society. Research considering the mechanism of the government anti-rumor spreading model is more practical significance.

The remainder of this paper is organized as follows. In Section 2, we derived the model considering the influence of rumors, after which a detailed steady-state analysis is carried out in Section 3, and the threshold of the rumor propagation is obtained. In Section 4, numerical simulation, uncertainty and sensitivity analysis of the model are presented. Finally, Section 5 summarizes this work.

\section{Model}

We assume that there are two kinds of spreaders in the whole population which called spreader with rumor and spreader with truth, respectively. As shown in Figure 1, the total population is divided into four different compartments name ignorants, spreaders with rumor, spreaders with truth and recovers, noted with $I(t), S_{+}(t), S_{-}(t), R(t)$ respectively. When an ignorant contacts a spreader with truth, the ignorant becomes the other side with rate $\beta_{+}$, otherwise, if he contacts a spread with rumor, the ignorant becomes the other party with rate $\beta_{-}$. When a spreader with truth contacts with spreader with rumor, they will become a 


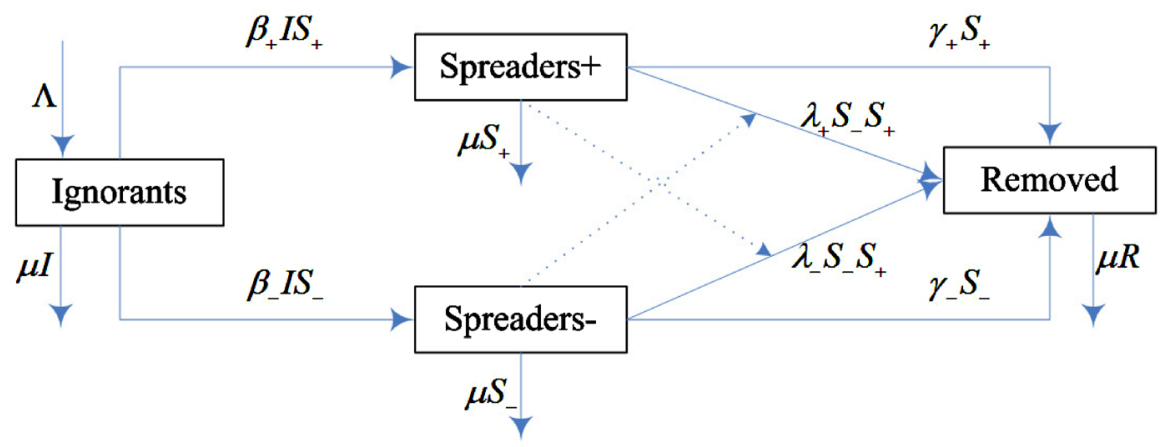

Figure 1. The flow diagram of the rumor propagation model.

removed with probability $\lambda_{+}$and $\lambda_{-}$. The spreaders $\left(S_{+} \backslash S_{-}\right)$spontaneously become the removed at a rate $\left(\gamma_{+} \backslash \gamma_{-}\right)$for losing interesting.

According to the dynamic interact as mentioned above, we established the S2IR rumor spreading model based on the above assumptions. The model is described as follows:

$$
\begin{aligned}
& \dot{I}=\Lambda-\beta_{-} S_{-} I-\beta_{+} S_{+} I-\mu_{1} I \\
& \dot{S}_{+}=\beta_{+} S_{+} I-\lambda_{+} S_{-} S_{+}-\gamma_{+} S_{+}-\mu_{2} S_{+} \\
& \dot{S_{-}}=\beta_{-} S_{-} I-\lambda \_S_{-} S_{+}-\gamma_{-} S_{-}-\mu_{3} S_{-} \\
& \dot{R}=\lambda_{+} S_{-} S_{+}+\lambda \_S_{-} S_{+}-\gamma_{-}+\gamma_{+} S_{+}+\gamma_{-} S_{-}-\mu_{4} R
\end{aligned}
$$

The parameters of the model descript as follows:

$\Lambda$, the constant recruitment rate of the population;

$\mu_{1}, \mu_{2}, \mu_{3}$, the natural fade away rate to recover of the ignorants, spreaders with rumor and spreaders with truth respectively;

$\beta_{+}, \beta_{-}$, the force of infection from ignorants to spreaders of rumor, spreaders of truth respectively;

$\lambda_{+}$, the fade away rate to recover when a spreader of rumor disseminate the rumor to a spreader of truth;

$\lambda_{-}$, the fade away rate to recover when a spreader of truth disseminate the truth to a spreader of rumor;

$\gamma_{+}, \gamma_{-}$, the forgetting rate of spreader of rumor and spreader of truth, respectively.

The initial condition of (1) is given as $I(0)>0, S_{+}(0) \geq 0, S_{-}(0) \geq 0, R(0) \geq 0$, for the fourth equation of system of (1) is not comprised in the first three equation. Then we can consider the simplified model as follows:

$$
\begin{aligned}
& \dot{I}=\Lambda-\beta_{-} S_{-} I-\beta_{+} S_{+} I-\mu_{1} I \\
& \dot{S}_{+}=\beta_{+} S_{+} I-\lambda_{+} S_{-} S_{+}-\gamma_{+} S_{+}-\mu_{2} S_{+} \\
& \dot{S}_{-}=\beta_{-} S_{-} I-\lambda S_{-} S_{+}-\gamma_{-} S_{-}-\mu_{3} S_{-}
\end{aligned}
$$

The initial condition of (2) is given as $I(0)>0, S_{+}(0) \geq 0, S_{-}(0) \geq 0$. Note $\mu=\max \left\{\mu_{1}, \mu_{2}, \mu_{3}\right\}$ Then we can easily verify that the system of (2) is mathematically well posed in the positive invariant region

$G=\left\{\left(I, S_{+}, S_{-}\right) \mid I>0, S_{+} \geq 0, S_{-} \geq 0, I+S_{+}+S_{-} \leq \Lambda / \mu\right\}$. And all the solutions 
with $I(0)>0, S_{+}(0) \geq 0, S_{-}(0) \geq 0$ approaches or enter the region $G$, so it is sufficient to consider solutions in $G$.

\section{Equilibria and Stability}

Noting that

$$
R_{0}=\max \left\{R_{01}, R_{02}\right\}
$$

where $R_{01}=\frac{\Lambda \beta_{+}}{\mu_{1}\left(\gamma_{+}+\mu_{2}\right)}, \quad R_{02}=\frac{\Lambda \beta_{-}}{\mu_{1}\left(\gamma_{-}+\mu_{3}\right)}$, and

$$
R^{*}=\min \left\{R_{1}^{*}, R_{2}^{*}\right\}
$$

where $R_{1}^{*}=\frac{\Lambda \beta_{+} \lambda_{-}+\beta_{+}\left(\mu_{2}+\gamma_{+}\right)\left(\mu_{3}+\gamma_{-}\right)}{\beta_{-}\left(\mu_{2}+\gamma_{+}\right)^{2}+\lambda_{-} \mu_{1}\left(\mu_{2}+\gamma_{+}\right)}$,

$$
R_{2}^{*}=\frac{\Lambda \beta_{-} \lambda_{+}+\beta_{-}\left(\mu_{3}+\gamma_{-}\right)\left(\mu_{2}+\gamma_{+}\right)}{\beta_{+}\left(\mu_{3}+\gamma_{-}\right)^{2}+\lambda_{+} \mu_{1}\left(\mu_{3}+\gamma_{-}\right)} \text {. }
$$

Letting the left-hand side of the differential equations of model (2) equal to zero yields the following equations

$$
\begin{aligned}
& 0=\Lambda-\beta_{-} S_{-} I-\beta_{+} S_{+} I-\mu_{1} I \\
& 0=\beta_{+} S_{+} I-\lambda_{+} S_{-} S_{+}-\gamma_{+} S_{+}-\mu_{2} S_{+} \\
& 0=\beta_{-} S_{-} I-\lambda_{-} S_{-} S_{+}-\gamma_{-} S_{-}-\mu_{3} S_{-}
\end{aligned}
$$

Obviously, the system exists a trivial equilibrium $E_{0}\left(I^{0}, 0,0\right)$ where $I^{0}=\Lambda / \mu_{1}$. If $R_{01}>1$, let $S_{-}=0$, we easily get the rumor-free equilibrium $E_{1}\left(I^{1}, S_{+}^{1}, 0\right)$ where $I^{1}=\left(\gamma_{+}+\mu_{2}\right) / \beta_{+}$, and

$S_{+}^{1}=\Lambda /\left(\gamma_{+}+\mu_{2}\right)-\mu_{1} / \beta_{+}=\frac{\mu_{1}}{\beta_{+}}\left(R_{01}-1\right)$. Similarly, If $R_{02}>1$, we get the counter-free equilibrium $E_{2}\left(I^{2}, 0, S_{-}^{2}\right)$ where $I^{2}=\left(\gamma_{-}+\mu_{3}\right) / \beta_{-}$, and $S_{-}^{2}=\Lambda /\left(\gamma_{-}+\mu_{3}\right)-\mu_{1} / \beta_{-}=\frac{\mu_{1}}{\beta_{-}}\left(R_{02}-1\right)$.

In the following, we shall study the existence of the positive equilibrium $E^{*}\left(I^{*}, S_{+}^{*}, S_{-}^{*}\right)$ of system (1). From the second and the third equation of (5), we have $S_{+}^{*}=\left(\beta_{-} I^{*}-\left(\mu_{3}+\gamma_{-}\right)\right) / \lambda_{-}, S_{-}^{*}=\left(\beta_{+} I^{*}-\left(\mu_{2}+\gamma_{+}\right)\right) / \lambda_{+}$.

Substituting them in the first equation of (5), we have

$$
F(I)=A\left(I^{*}\right)^{2}+B I^{*}-C
$$

where $A=\beta_{+} \beta_{-}\left(\lambda_{+}+\lambda_{-}\right), \quad B=\left(\lambda_{+}+\lambda_{-}\right) \mu_{1}-\beta_{+} \lambda_{+}\left(\mu_{3}+\gamma_{-}\right)-\beta_{-} \lambda_{-}\left(\mu_{2}+\gamma_{+}\right)$, and $C=\Lambda \lambda_{+} \lambda_{-}$. Obviously, $A>0, C>0$ then exist a unique positive solution $I=I^{*}$, yield $F\left(I^{*}\right)=0$. For the $S_{+}^{*}>0$ and $S_{-}^{*}>0$, we have $F\left(\left(\mu_{2}+\gamma_{+}\right) / \beta_{+}\right)<0$ and $F\left(\left(\mu_{3}+\gamma_{-}\right) / \beta_{-}\right)<0$

From (6) we get that $R_{1}^{*}>1, R_{2}^{*}>1$, namely $R^{*}>0$. Thus, we obtain the following theorem.

Theorem 1. System (2) has the following equilibrium:

1) if $R_{0}<1$, system (2) has only the trivial equilibrium $E_{0}$;

2) if $R_{01}>1, R_{02}<1$, system (2) has a trivial equilibrium $E_{0}$ and a 
truth-free equilibrium $E_{1}$;

3) if $R_{01}<1, R_{02}>1$, system (2) has a trivial equilibrium $E_{0}$ and a rumor-free equilibrium $E_{2}$;

4) if $R_{01}<1, R_{02}>1$, system (2) has a trivial equilibrium $E_{0}$, a truth-free equilibrium $E_{1}$ and a rumor-free equilibrium $E_{2}$;

5) if $R_{01}>1, R_{02}>1, R^{*}>1$, system (2) has a trivial equilibrium $E_{0}$, a truth-free equilibrium $E_{1}$, a rumor-free equilibrium $E_{2}$ and coexist equilibrium $E^{*}$.

The general Jacobian of (2) is given by

$$
J_{(2.2)}=\left|\begin{array}{ccc}
-\beta_{+} S_{+}-\beta_{-} S_{-}-\mu_{1} & -\beta_{+} I & -\beta_{-} I \\
\beta_{+} S_{+} & \beta_{+} I-\lambda_{+} S_{-}-\gamma_{+}-\mu_{2} & -\lambda_{+} S_{+} \\
\beta_{-} S_{-} & -\lambda_{-} S_{-} & \beta_{-} I-\lambda_{-} S_{+}-\gamma_{-}-\mu_{3}
\end{array}\right|
$$

Theorem 2. The boundary equilibrium points of (1) have the following local stability properties:

1) if $R_{0}<1, E_{0}\left(I_{0}, 0,0\right)$ is a stable node, and if $R_{0}>1, E_{0}\left(I_{0}, 0,0\right)$ is a saddle node.

2) $E_{1}\left(I_{1}, S_{+}^{1}, 0\right)$ is a stable if and only if $R_{1}^{*}>1$.

3) $E_{2}\left(I_{1}, 0, S_{-}^{2}\right)$ is a stable if and only if $R_{2}^{*}>1$.

4) $E^{*}\left(I^{*}, S_{+}^{*}, S_{-}^{*}\right)$ is always unstable if it exists.

Proof. 1) At $E_{0}$, we have

$$
J_{(2.2)}\left|E_{0}=\right| \begin{array}{ccc}
-\mu_{1} & -\frac{\Lambda \beta_{+}}{\mu_{1}} & -\frac{\Lambda \beta_{-}}{\mu_{1}} \\
0 & \frac{\Lambda \beta_{+}}{\mu_{1}}-\left(\gamma_{+}+\mu_{2}\right) & 0 \\
0 & 0 & \frac{\Lambda \beta_{-}}{\mu_{1}}-\left(\gamma_{-}+\mu_{3}\right)
\end{array} \mid
$$

The eigenvalues of the Equation (8) are $\lambda_{1}=-\mu_{1}$,

$\lambda_{2}=\left(\Lambda \beta_{+}-\mu_{1}\left(\gamma_{+}+\mu_{2}\right)\right) / \mu_{1}=\left(\gamma_{+}+\mu_{2}\right) /\left(R_{01}-1\right)$,

$\lambda_{3}=\left(\Lambda \beta_{-}-\mu_{1}\left(\gamma_{-}+\mu_{3}\right)\right) / \mu_{1}=\left(\gamma_{-}+\mu_{3}\right) /\left(R_{02}-1\right)$, and therefore the $E_{0}$ is an unstable node if $R_{0}<1$.

2) At $E_{1}$, the Jacobian of (2) is

$$
J_{(2.2)}\left|E_{1}=\right| \begin{array}{ccc}
-\frac{\Lambda \beta_{+}}{\gamma_{+}+\mu_{2}} & -\left(\gamma_{+}+\mu_{2}\right) & -\frac{\beta_{-}\left(\gamma_{+}+\mu_{2}\right)}{\beta_{+}} \\
\frac{\Lambda \beta_{+}}{\gamma_{+}+\mu_{2}}-\mu_{1} & 0 & -\frac{\left(\Lambda \beta_{1}-\mu_{1}\left(\lambda_{+}+\mu_{2}\right)\right) \gamma_{+}}{\beta_{+}\left(\lambda_{+}+\mu_{2}\right)} \\
0 & 0 & \frac{\left(R_{1}^{*}-1\right)\left(\beta_{-}\left(\lambda_{+}+\mu_{2}\right)+\gamma_{-} \mu_{1}\right)}{\beta_{+}}
\end{array} \mid
$$

The eigenvalues are $\lambda_{1}=\left(1-R_{1}^{*}\right)\left(\beta_{+}\left(\gamma_{-}+\mu_{3}\right)\right) / \beta_{2}$,

$\lambda_{2,3}=-\frac{\mu_{1}\left(R_{01} \pm \sqrt{R_{01}^{2}-\left(R_{01}-1\right)\left(\lambda_{+}+\mu_{2}\right) / \mu_{1}}\right.}{2}$, obviously they all have negative 
real parts and $E_{1}$ is local stability if $R_{1}^{*}>1$ and $R_{01}>1$.

In a similar fashion, we can conclude that if $R_{2}^{*}>1$ and $R_{02}>1 . E_{2}$ is local stability.

For the positive equilibrium $E^{*}$,

$$
\left|\begin{array}{ccc}
\frac{\Lambda^{*}}{I} & -\beta_{+} I^{*} & -\beta_{-} I^{*} \\
\frac{\beta_{-} I^{*}-\left(\lambda_{-}+\mu_{3}\right) \beta_{+}}{\beta_{-}} & 0 & -\frac{\left(\beta_{-} I^{*}-\left(\lambda_{-}+\mu_{3}\right)\right) \gamma_{+}}{\beta_{-}} \\
\frac{\left(\beta_{+} I^{*}-\left(\lambda_{+}+\mu_{2}\right)\right) \beta_{-}}{\beta_{+}} & -\frac{\left(\beta_{+} I^{*}-\left(\lambda_{+}+\mu_{2}\right)\right) \gamma_{-}}{\beta_{+}} & 0
\end{array}\right|
$$

The characteristic polynomial of (10) can be calculated as follows is

$$
\lambda^{3}+\frac{\Lambda}{I^{*}} \lambda^{2}+\left(\beta_{+}^{2} S_{+}^{*} I^{*}+\beta_{-}^{2} S_{-}^{*} I^{*}-\lambda_{+} \lambda_{-} S_{+}^{*} S_{-}^{*}\right) \lambda-f=0
$$

which $f=\left(\beta_{+} \beta_{-}\left(\lambda_{+}+\lambda_{-}\right) S_{+}^{*} S_{-}^{*}\right)+\frac{\Lambda S_{+}^{*} S_{-}^{*} \lambda_{+} \lambda_{-}}{I^{*}}$, the eigenvalues satisfied $\lambda_{1} \lambda_{2} \lambda_{3}=f>0$, It can be obtained by the Routh-Hurwitz criterion: It exists at least one eigenvalue of the polynomial equation has positive real part, then $E^{*}$ is always unstable. $\square$

Next, we will consider the global stability of quiet equilibrium $E_{0}$ of system (2).

Theorem 3. The quiet equilibrium $E_{0}=\left(I_{0}, 0,0\right)$ is globally asymptotically stable provided that $R_{0}<1$.

Proof. Define the Lyapunov function

$$
V\left(I, S_{+}, S_{-}\right)=V_{1}\left(I, S_{+}, S_{-}\right)+V_{2}\left(I, S_{+}, S_{-}\right)
$$

where $V_{1}\left(I, S_{+}, S_{-}\right)=I-I_{0}-I_{0} \ln \frac{I}{I}, V_{2}\left(I, S_{+}, S_{-}\right)=S_{+}+S_{-}$, then differentiating $V_{1}$ with respect to $t$ along solutions of system (2) gives

$$
\begin{aligned}
& \frac{\mathrm{d} V_{1}}{\mathrm{~d} t}=\left(1-\frac{I_{0}}{I}\right)\left(\Lambda-\beta_{-} S_{-} I-\beta_{+} S_{+} I-\mu_{1} I\right) \\
&=\left(1-\frac{I_{0}}{I}\right)\left(-\beta_{-} S_{-} I-\beta_{+} S_{+} I\right)+\mu_{1} I_{0}\left(1-\frac{I_{0}}{I}\right)\left(1-\frac{I}{I_{0}}\right) \\
& \leq\left(1-\frac{I_{0}}{I}\right)\left(-\beta_{-} S_{-} I-\beta_{+} S_{+} I\right) \\
&=-\beta_{-} S_{-} I-\beta_{+} S_{+} I+\beta_{-} S_{-} I_{0}+\beta_{+} S_{+} I_{0} \\
& \frac{\mathrm{d} V_{2}}{\mathrm{~d} t}=\beta_{+} S_{+} I-\lambda_{+} S_{-} S_{+}-\gamma_{+} S_{+}-\mu_{2} S_{+}+\beta_{-} S_{-} I-\lambda_{-} S_{-} S_{+}-\gamma_{-} S_{-}-\mu_{3} S_{-}
\end{aligned}
$$

Then, we have

$$
\begin{aligned}
\frac{\mathrm{d} V}{\mathrm{~d} t} & =\frac{\mathrm{d} V_{1}}{\mathrm{~d} t}+\frac{\mathrm{d} V_{2}}{\mathrm{~d} t} \\
& \leq \beta_{+} S_{+} I_{0}-\lambda_{+} S_{-} S_{+}-\left(\gamma_{+}+\mu_{2}\right) S_{+}+\beta_{-} S_{-} I_{0}-\lambda_{-} S_{-} S_{+}-\left(\gamma_{-}-\mu_{3}\right) S_{-} \\
& =\left(\gamma_{+}+\mu_{2}\right)\left(R_{01}-1\right) S_{+}+\left(\gamma_{-}+\mu_{3}\right)\left(R_{02}-1\right) S_{-}-\left(\lambda_{+}+\lambda_{-}\right) S_{-} S_{+} \\
& \leq\left(\gamma_{+}+\mu_{2}\right)\left(R_{0}-1\right) S_{+}+\left(\gamma_{-}+\mu_{3}\right)\left(R_{0}-1\right) S_{-}-\left(\lambda_{+}+\lambda_{-}\right) S_{-} S_{+}
\end{aligned}
$$


Therefore, when $R_{0}<1, \frac{\mathrm{d} V}{\mathrm{~d} t} \leq 0$, and the equality holds only for $I=I_{0}$, $S_{+}=0$ and $S_{-}=0$, According to the LaSalle invariant principle, we have $\lim _{t \rightarrow \infty} I(t)=I_{0}, \quad \lim _{t \rightarrow \infty} S_{+}(t)=0$ and $\lim _{t \rightarrow \infty} S_{-}(t)=0$. Thus, the proof is completed.

\section{Numerical Studies}

\subsection{Numerical Simulation}

We have formulated a S2IR rumor-spread model. We have established some threshold conditions for the steady state. To illustrate the analytical results, we do some numerical simulations. From Table 1 , we can set $\mu_{1}=0.3, \mu_{2}=0.3$, $\mu_{3}=0.3, \gamma_{+}=0.4, \gamma_{-}=0.4, \lambda_{+}=0.38, \lambda_{-}=0.1$, then system (2) becomes

$$
\begin{aligned}
& \dot{I}=\Lambda-\beta_{-} S_{-} I-\beta_{+} S_{+} I-0.3 I \\
& \dot{S}_{+}=\beta_{+} S_{+} I-0.38 S_{-} S_{+}-0.4 S_{+}-0.3 S_{+} \\
& \dot{S}_{-}=\beta_{-} S_{-} I-0.1 S_{-} S_{+}-0.4 S_{-}-0.3 S_{-}
\end{aligned}
$$

Let $\Lambda=0.2, \beta_{+}=0.705, \beta_{-}=0.725$, then $R_{0}=0.6905<1$. By theorem 1.(a), theorem 2.(a) and theorem 3 , system (16) has only the trivial equilibrium $E_{0}(0.6667,0,0)$ and it is globally asymptotically stable (see Figure 2$)$, it means that the message(rumor or truth) will eliminate.

Let $\Lambda=0.312, \quad \beta_{+}=0.856, \quad \beta_{-}=0.656$. We have $R_{01}=1.272>1$, $R_{02}=0.974<1, R_{1}^{*}=1.303>1, R_{2}^{*}=0.7996<1$, It presents the system $(16)$ has have two equilibria, $E_{0}(1.04,0,0)$ and $E_{1}(0.8178,0.095,0)$ where $E_{0}$ is unstable but $E_{1}$ is stable according to theorem 1.(b) and theorem 2.(a)-2.(b) (see Figure $3(\mathrm{a}))$. Let $\Lambda=0.323, \beta_{+}=0.6215, \beta_{-}=0.8545$, then system $R_{01}=0.956<1$, $R_{02}=1.314>1, R_{1}^{*}=0.738<1, R_{2}^{*}=1.362>1$. It shows that the system has

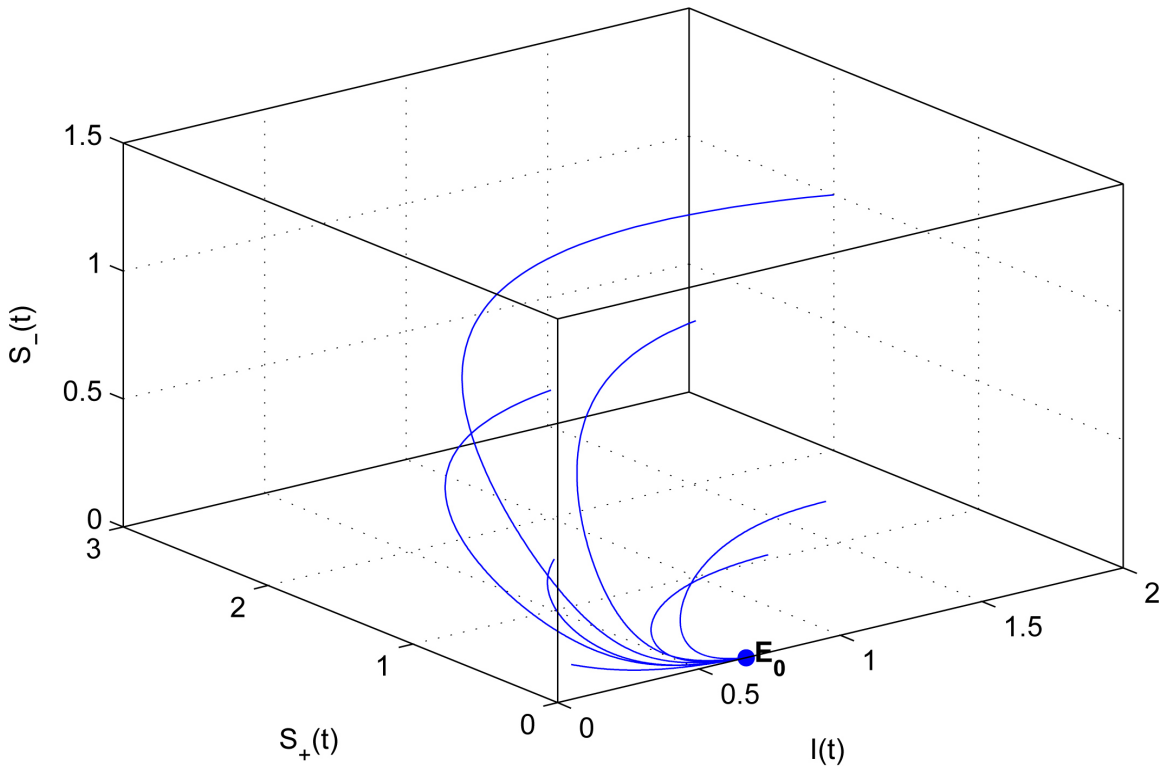

Figure 2. The trajectories, equilibria and stability of system (4.1) with $\Lambda=0.2, \beta_{+}=0.705, \beta_{-}=0.725$. 


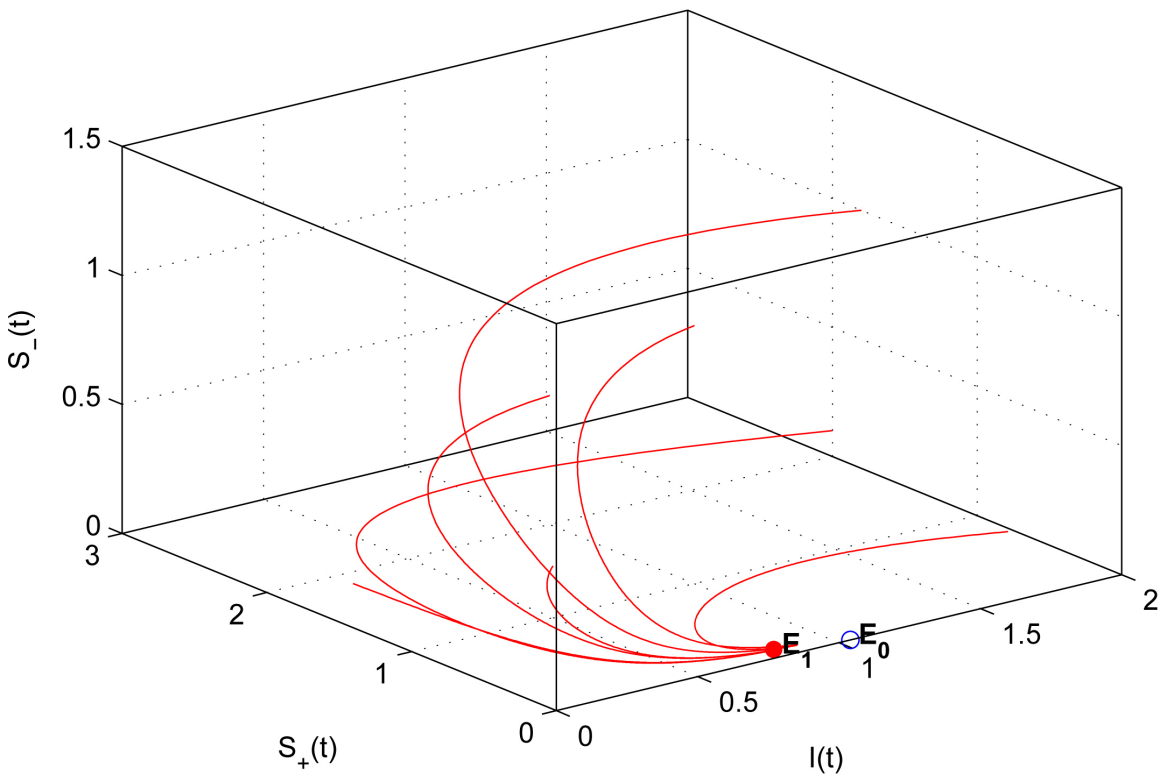

(a)

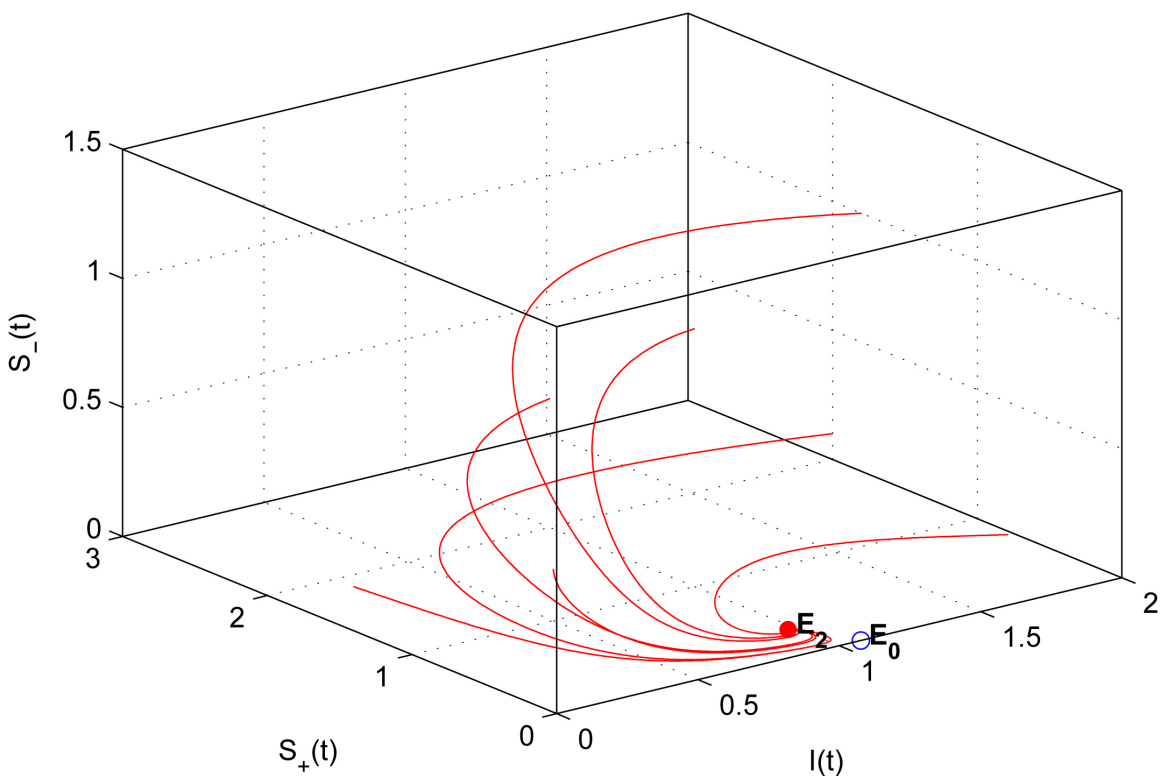

(b)

Figure 3. the trajectories, equilibria and stability of system (16) with (a) $\Lambda=0.323, \beta_{+}=0.6215, \beta_{-}=0.8545$, (b) $\Lambda=0.323, \beta_{+}=0.6215, \beta_{-}=0.8545$.

two equilibria, $E_{0}(1.077,0,0)$ and $E_{2}(0.819,0,0.11)$ where $E_{0}$ is unstable but $E_{2}$ is stable according to theorem 1.c and theorem 2.a, 2.c (see Figure 3(b)).

Let $\Lambda=2.1, \beta_{+}=0.6, \beta_{-}=0.9$, then $R_{0}=9>0, R_{1}^{*}=0.9091<1$, $R_{2}^{*}=3.101>1$. By theorem 1.(d), system (16) has three equilibria $E_{0}(7,0,0)$, $E_{1}(1.167,2.5,0)$ and $E_{2}(0.778,0,2.667)$ and $E_{0}, E_{1}$ is unstable, but $E_{2}$ is stable (see Figure $4(\mathrm{a})$ ). Let $\Lambda=2.8, \beta_{+}=0.76, \beta_{-}=0.72$, then $R_{0}=10.13>0, R^{*}=R_{1}^{*}=1.566>1, R_{2}^{*}=2.474>1$. By theorem 1.(e), system (16) has three equilibria $E_{0}(9.33,0,0), E_{1}(0.921,3.605,0), E_{2}(0.972,0,3.583)$ and $E^{*}(1.244,1.956,0.646)$, which $E_{0}$ and $E^{*}$ are not stable but $E_{1}$ and 


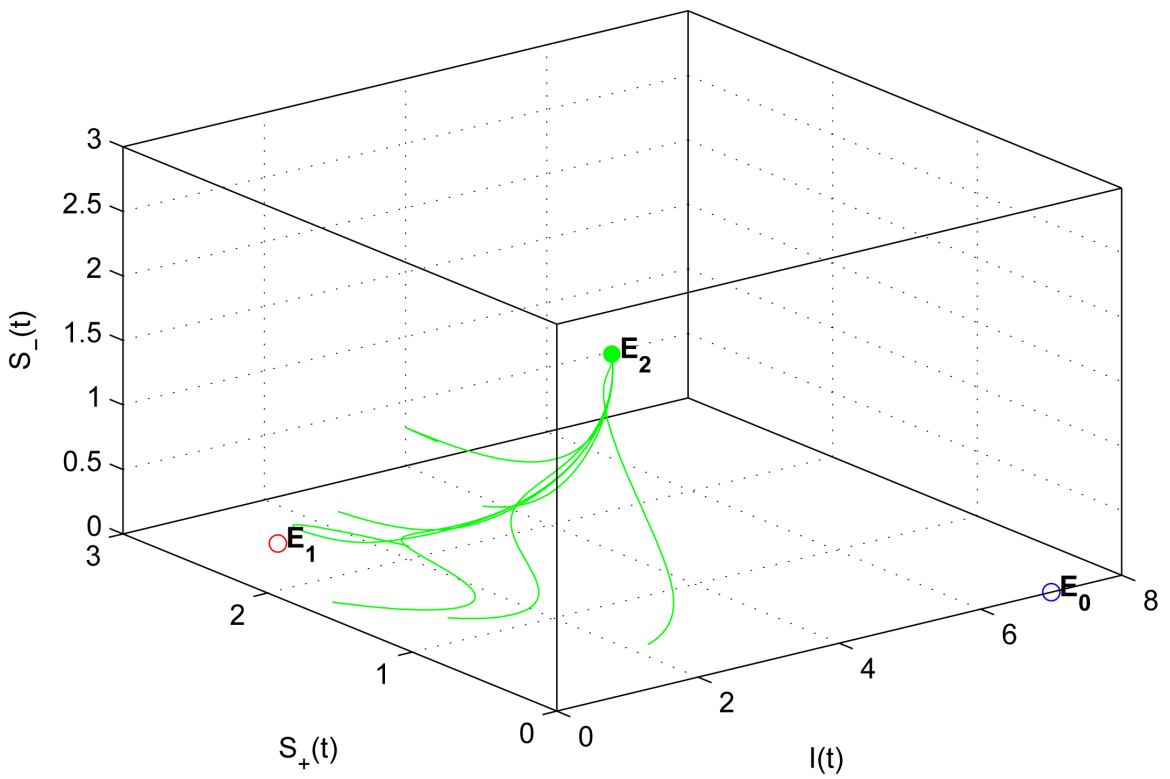

(a)

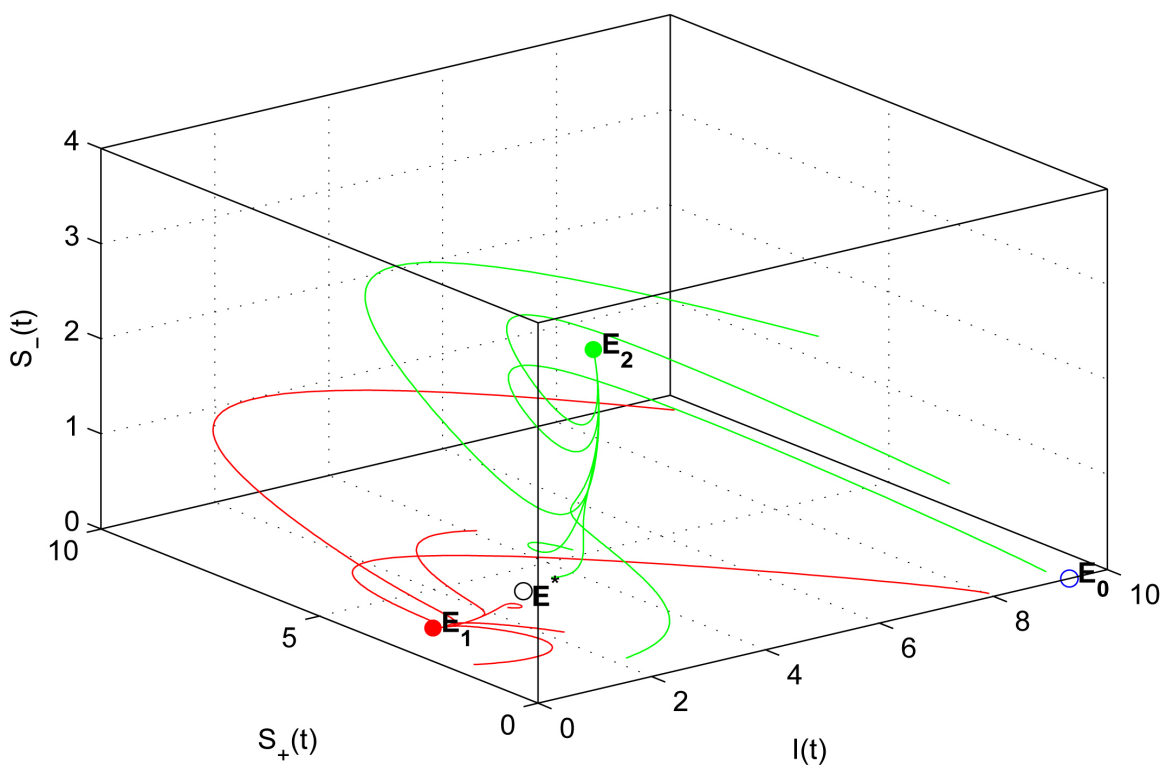

(b)

Figure 4. The trajectories, equilibria and stability of system (16) with (a) $\Lambda=2.1, \beta_{+}=0.6, \beta_{-}=0.9$ (b) $\Lambda=2.8, \beta_{+}=0.76, \beta_{-}=0.72$.

$E_{2}$ are bistable. The phase portrait is represented in Figure 4(b).

\subsection{Uncertainty and Sensitivity Analysis}

In this part, uncertainty and sensitivity analysis, based on Latin hypercube sampling (LHS) and partial rank correlation coefficients (PRCC) scheme, will explore the dependence of $R_{0}, R_{1}^{*}$ and $R_{2}^{*}$ due to the change of input parameters in the estimation of the uncertainty. Each input parameters is sampling 1000 times. A uniform distribution function was used and the variation ranges of all parameters are given in Table 1. 
Table 1. Input parameter sample values for simulation.

\begin{tabular}{ccccc}
\hline Descriptions & Symbols & Values (range) & Reference \\
\hline The constant recruitment rate of the population & $\Lambda$ & $1.5(0.2,2.8)$ & Assumed \\
The natural fade away rate of the ignorants & $\mu_{1}$ & $0.5(0.1,0.9)$ & {$[29]$} \\
The natural fade away rate of spreaders with rumor & $\mu_{2}$ & $0.5(0.1,0.9)$ & {$[29]$} \\
The natural fade away rate of spreaders with truth & $\mu_{3}$ & $0.5(0.1,0.9)$ & {$[29]$} \\
The force of infection from ignorants to rumor-monger & $\beta_{+}$ & $0.8(0.6,1)$ & {$[30]$} \\
The force of infection from ignorants to truth-spreader & $\beta_{-}$ & $0.8(0.6,1)$ & {$[30]$} \\
The fade away rate of rumor-mongers & $\lambda_{+}$ & $0.2(0.01,0.39)$ & Assumed \\
The fade away rate of truth-spreaders & $\lambda_{-}$ & $0.2(0.01,0.39)$ & Assumed \\
The forgetting rate of spreaders of rumor & $\gamma_{+}$ & $0.3(0.1,0.5)$ & {$[30]$} \\
The forgetting rate of spreaders of truth & $\gamma_{-}$ & $0.3(0.1,0.5)$ & {$[30]$} \\
\hline
\end{tabular}

Table 2. Input parameter sample values for simulation.

\begin{tabular}{ccccccc}
\hline \multirow{2}{*}{ Parameter } & \multicolumn{2}{c}{$R_{0}$} & \multicolumn{2}{c}{$R_{1}^{*}$} & \multicolumn{2}{c}{$R_{2}^{*}$} \\
\cline { 2 - 7 } & PRCC & p-value & PRCC & p-value & PRCC & p-value \\
\hline$\Lambda$ & 0.0867 & 0.0064 & 0.2224 & 0 & 0.3448 & 0 \\
$\mu_{1}$ & -0.0379 & 0.2348 & -0.0601 & 0.0592 & -0.1306 & 0 \\
$\mu_{2}$ & 0.0186 & 0.5602 & -0.7155 & 0 & 0.4062 & 0 \\
$\mu_{3}$ & -0.0751 & 0.0183 & 0.3442 & 0 & -0.7685 & 0 \\
$\beta_{+}$ & -0.0378 & 0.2349 & 0.5305 & 0 & -0.5457 & 0 \\
$\beta_{-}$ & 0.0721 & 0.0235 & -0.489 & 0 & 0.6155 & 0 \\
$\lambda_{+}$ & 0 & 0 & -0.1302 & 0 & 0.772 & 0 \\
$\lambda_{-}$ & 0 & 0 & 0.7415 & 0 & -0.1256 & 0.0001 \\
$\gamma_{+}$ & 0.038 & 0.2324 & -0.6316 & 0 & 0.3833 & 0 \\
$\gamma_{-}$ & -0.0695 & 0.0291 & 0.3296 & 0 & -0.6966 & 0 \\
\hline
\end{tabular}

The PRCC results which illustrate the dependence on each parameters of $R_{0}$, $R_{1}, R_{2}$ respectively. We considered $|\mathrm{PRCC}| \geq 0.4$ as indicating that the high correlation between input parameters and output variables, $0.2 \leq|\mathrm{PRCC}|<0.4$ as moderate correlations and $|\mathrm{PRCC}|<0.2$ as no relations.

The results of the simulation of PRCCs are shown in Table 2 and Figure 5. For $R_{0}$, the absolute values of PRCCs of each parameters fall below 0.2. It suggests that the impact of $R_{0}$ is the result of the interaction of each parameters and it is very difficult to decrease $R_{0}$ by regulating and controlling a few parameters. The parameters with the most positive impact on $R_{1}$ are the fade away rate of truth-spreaders $\lambda_{-}$and the force of infection of rumor-spreaders $\beta_{+}$, while the most negative impact on $R_{1}$ are the natural fade away of truth-spreaders $\mu_{2}$, the forgetting rate of rumor-spreader $\gamma_{+}$and the force of infection of truth-spreader $\beta_{-}$. It means that the five factors play a critical role 
on the process of rumor-spreading. The parameters that have the moderate influence with $R_{1}$ are the recruitment $\Lambda$, the natural fade away of truth-spreader $\mu_{3}$ and the forgetting rate of truth-spreader $\gamma_{-}$. Compare with $R_{2}$, we find the parameters $\beta_{+}, \beta_{-}, \mu_{2}, \mu_{3}, \gamma_{+}, \gamma_{-}$have opposite influences on $R_{1}$ and $R_{2}$. This exhibitions the propagation have the nature of competitive exclusion between rumor with truth. The parameters of the fade away rate $\lambda_{+}, \lambda_{-}$have no relations with $R_{1}, R_{2}$ respectively, but have high positive relations with $R_{2}, R_{1}$ respectively. If both $\lambda_{+}$and $\lambda_{-}$are large, it is possible that both $R_{1}$ and $R_{2}$ are greater than 1 and this will lead to the occurrence of bistable on $E_{1}$ and $E_{2}$.

Figures 6(a)-(c) shows the LHSs frequency distributions. The mean value for $R_{0}, R_{1}$ and $R_{2}$ are $M_{R_{0}}=2.4214, M_{R_{1}}=1.4797, M_{R_{2}}=1.4793$ respectively. The standard deviation with $R_{0}$ is 0.6642 and greater than with $R_{1}$ and $R_{2} \quad(0.3120,0.3037$ respectively) which indicates that the derived frequency distribution for $R_{0}$ is dispersed than the distributions for $R_{1}$ and $R_{2}$.

\section{Conclusion}

Rumor propagation has been investigated through different types of mathematical models. In our study, we consider a rumor propagation model with truth-spreading and determine the threshold which governs the dynamics of the system. On one hand, the propagation of rumor and truth is mutually

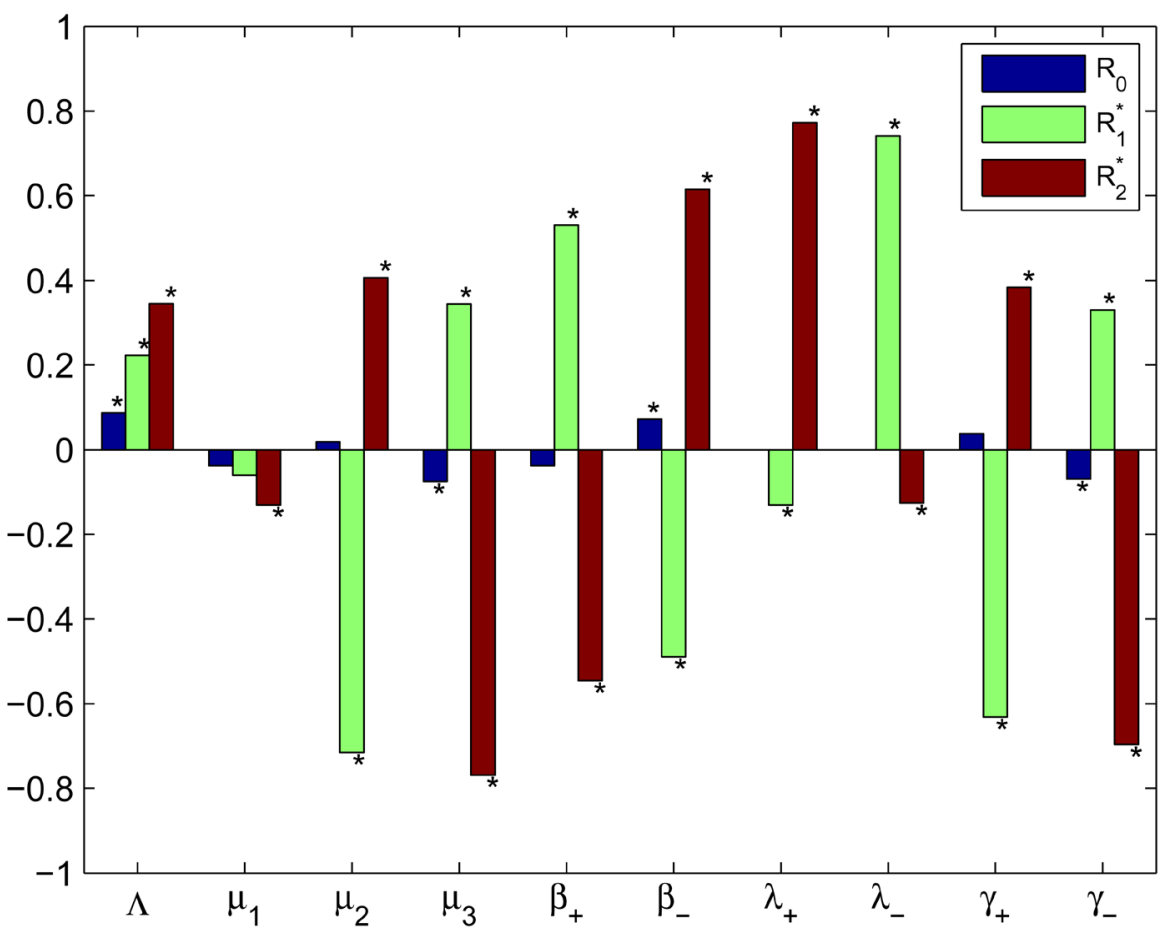

Figure 5. PRCCs illustrating the dependence of $R_{0}, R_{1}^{*}$ and $R_{2}^{*}$ for the rumor spread model on each parameter. Symbol * shows the PRCC value is not zero significantly $(P<0.05)$. 
(a)
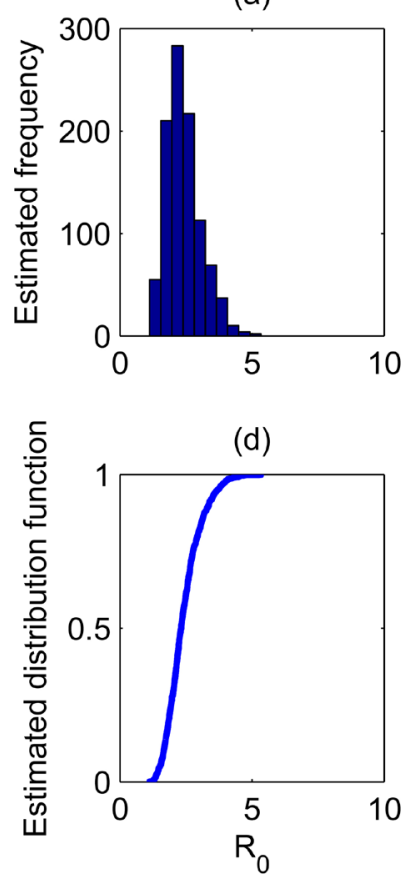

(b)

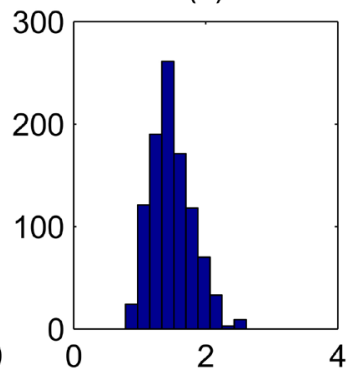

(e)

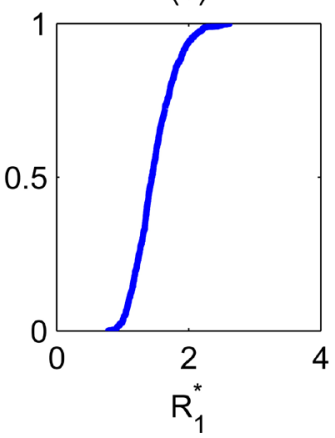

(c)

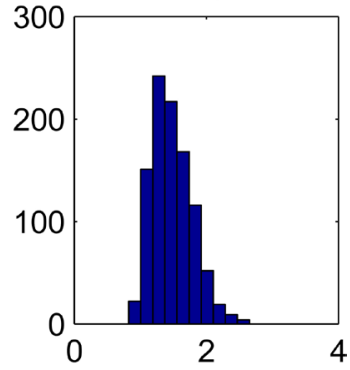

(f)

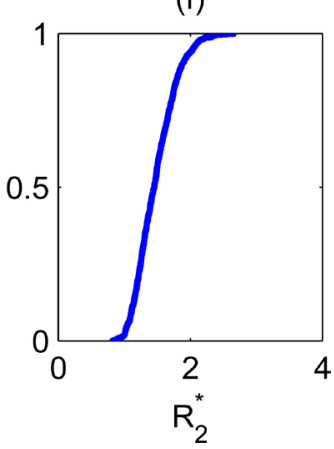

Figure 6. Uncertainty results based on Latin hypercube sampling. The top row consists of the frequency diagrams for (a) $R_{0}$, (b) $R_{1}^{*}$ and (c) $R_{2}^{*}$. The following row shows estimates of CDFs for output variables ((d) $R_{0}$, (e) $R_{1}^{*}$ and (f) $R_{2}^{*}$ ).

exclusive. Therefore, the appropriate increase in the spread of truth is conducive to the elimination of the spread of rumors. On the other hand, the spread of rumor and truth can coexist for a long time under certain conditions. At this point, the improvement of the spread of the truth is also conducive to the spread of rumors in a certain range. This requires the government and related organizations to further develop the corresponding work.

\section{Acknowledgements}

The research has been supported by The Social Science Planning Projects of Jiangxi Province (14XW08) and The 12th Five-year Education Scientific Planning Project of Jiangxi Province (15ZD3LYB031).

\section{References}

[1] Zhu, H. (2010) Psychological Impact of Rumors of Public Health Emergencies on Hainan People. Medicine \& Society, 23, 15-17.

[2] Steele, T.J., Smith, S.M. and Mcbroom, W.H. (1999) Consumer Rumors and Corporate Communications: Rumor Etiology, Background, and Potential Devastating Consequences. Journal of Marketing Management, 9, 95-97.

[3] Wakamatsu, H. and Miyata, T. (2014) Impacts of Actual Harm and Harmful Rumors from Radioactive Spill from the Fukushima Disaster on the Japanese Seafood Market. International Institute of Fisheries Economics \& Trade.

[4] Kermack, W.O. and Mckendrick, A.G. (1991) Contributions to the Mathematical Theory of Epidemics-I. Bulletin of Mathematical Biology, 53, 89-118. 
[5] Li, M.Y., Graef, J.R., Wang, L., et al. (1999) Global Dynamics of a Seir Model with Varying Total Population Size. Mathematical Biosciences, 160, 191-213. https://doi.org/10.1016/S0025-5564(99)00030-9

[6] Li, M.Y. and Muldowney, J.S. (1999) Global Stability for the Seir Model in Epidemiology. Mathematical Biosciences, 125, 155-164. https://doi.org/10.1016/0025-5564(95)92756-5

[7] Wang, N., Pang, J. and Wang, J. (2014) Stability Analysis of a Multigroup Seir Epidemic Model with General Latency Distributions. Abstract \& Applied Analysis, 2014, Article ID: 740256. https://doi.org/10.1155/2014/740256

[8] Egbetade, S.A. and Ibrahim, M.O. (2013) Stability Analysis of Equilibrium States of an Seir Tuberculosis Model. Journal of the Nigerian Association of Mathematical Physics, 20, 119-124.

[9] Tipsri, S. and Chinviriyasit, W. (2014) Stability Analysis of Seir Model with Saturated Incidence and Time Delay. IJAPM, 4, 42-45. https://doi.org/10.7763/IJAPM.2014.V4.252

[10] Meng, F., Li, M.Y. and Ke, W. (2001) Global Stability of an Seis Epidemic Model with Recruitment and a Varying Total Population Size. Mathematical Biosciences, 170, 199-208. https://doi.org/10.1016/S0025-5564(00)00067-5

[11] Liu, W.M. and van den Driessche, P. (1995) Epidemiological Models with Varying Population Size and Dose-Dependent Latent Period. Mathematical Biosciences, 128, 57-69. https://doi.org/10.1016/0025-5564(94)00067-A

[12] Greenhalgh, D. (1997) Hopf Bifurcation in Epidemic Models with a Latent Period and Nonpermanent Immunity. Mathematical \& Computer Modelling, 25, 85-107. https://doi.org/10.1016/S0895-7177(97)00009-5

[13] San-Ling, Y., Han, L.T. and Zhi-En, M.A. (2001) A Kind of Epidemic Model Having Infectious Force in Both Latent Period and Infected Period. Journal of Biomathematics, 16, 392-398.

[14] Zhang, T. and Teng, Z. (2007) On a Nonautonomous Seirs Model in Epidemiology. Bulletin of Mathematical Biology, 69, 2537-2559. https://doi.org/10.1007/s11538-007-9231-z

[15] Daley, D.J. and Kendall, D.G. (1964) Epidemics and Rumours. Nature, 204, 1118. https://doi.org/10.1038/2041118a0

[16] Daley, D.J. and Kendall, D.G. (1965) Stochastic Rumours. IMA Journal of Applied Mathematics, 1, 42-55. https://doi.org/10.1093/imamat/1.1.42

[17] Murray, J.D. (1980) Mathematical Modelling in Epidemiology.

[18] Maki, D.P. and Thompson, M. (1973) Mathematical Models and Applications.

[19] Zanette, D.H. (2001) Critical Behavior of Propagation on Small-World Networks. Physical Review E Statistical Nonlinear \& Soft Matter Physics, 64, 1725-1732. https://doi.org/10.1103/PhysRevE.64.050901

[20] Zanette, D.H. (2002) Dynamics of Rumor Propagation on Small-World Networks. Physical Review E, 65, 110-126. https://doi.org/10.1103/PhysRevE.65.041908

[21] Bartolozzi, M., Leinweber, D.B. and Thomas, A.W. (2005) Stochastic Opinion Formation in Scale-Free Networks. Physical Review E Statistical Nonlinear \& Soft Matter Physics, 72, 138-148. https://doi.org/10.1103/PhysRevE.72.046113

[22] Simas, T. and Rocha, L.M. (2009) Stochastic Model for Scale-Free Networks with Cutoffs. Physical Review E Statistical Nonlinear \& Soft Matter Physics, 78, 837-849.

[23] Isham, V., Harden, S. and Nekovee, M. (2010) Stochastic Epidemics and Rumours on Finite Random Networks. Physica A Statistical Mechanics \& Its Applications, 
389, 561-576. https://doi.org/10.1103/PhysRevE.72.046113

[24] Hou, Y., Xie, H. and Ma, J. (2013) Stochastic Model of Rumor Spreading in Scale-Free Networks. International Journal of Advancements in Computing Technology, 5, 376-384. https://doi.org/10.4156/ijact.vol5.issue9.45

[25] Kawachi, K. (2008) Deterministic Models for Rumor Transmission. Nonlinear Analysis Real World Applications, 9, 1989-2028. https://doi.org/10.4156/ijact.vol5.issue9.45

[26] Xia, L.L., Jiang, G.P., Song, B., et al. (2015) Rumor Spreading Model Considering Hesitating Mechanism in Complex Social Networks. Physica A Statistical Mechanics \& Its Applications, 437, 295-303. https://doi.org/10.1016/j.physa.2015.05.113

[27] Zan, Y., Wu, J., Li, P., et al. (2014) Sicr Rumor Spreading Model in Complex Networks: Counterattack and Self-Resistance. Physica A Statistical Mechanics \& Its Applications, 405, 159-170. https://doi.org/10.1016/j.physa.2014.03.021

[28] Gu, J., Li, W. and Cai, X. (2008) The Effect of the Forget-Remember Mechanism on Spreading. European Physical Journal B, 62, 247-255. https://doi.org/10.1140/epjb/e2008-00139-4

[29] Zhao, L., Wang, Q., Cheng, J., et al. (2011) Rumor Spreading Model with Consideration of Forgetting Mechanism: A Case of Online Blogging Live Journal. Physica A Statistical Mechanics \& Its Applications, 390, 2619-2625. https://doi.org/10.1016/j.physa.2011.03.010

[30] Zhao, L., Qiu, X., Wang, X., et al. (2013) Rumor Spreading Model Considering Forgetting and Remembering Mechanisms in Inhomogeneous Networks. Physica $A$ Statistical Mechanics \& Its Applications, 392, 987-994. https://doi.org/10.1016/j.physa.2012.10.031

[31] Wang, J., Zhao, L. and Huang, R. (2014) 2SI2R Rumor Spreading Model in Homogeneous Networks. Physica A Statistical Mechanics \& Its Applications, 413, 153-161. https://doi.org/10.1016/j.physa.2014.06.053 